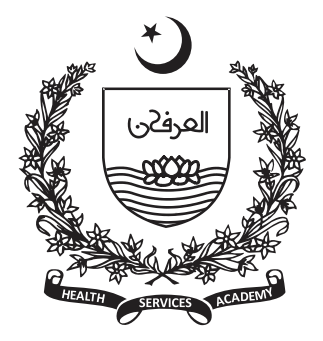

${ }^{1}$ Assistant Professor, department of Pulmonology, Saidu Group of Teaching Hospital Swat.

${ }^{2}$ Assistant Professor Medicine ICU, Saidu Group of Teaching Hospital Swat.

${ }^{3} \mathrm{RN}, \mathrm{BSN}, \mathrm{MSPH}$, Swat College of Nursing, RN, Saidu Group of Teaching Hospital Swat

${ }^{4}$ District Specialist, department of Pulmonology, Saidu Group of Teaching Hospital Swat. 5Principal, Swat College of Nursing, Swat.

${ }^{6}$ Trainee Medical officer, Saidu Group of Teaching Hospital Swat.

${ }^{7}$ Assistant Professor, Anaesthesiology, Saidu Group of Teaching Hospital Swat. ${ }^{8}$ Medical Superintendent, Saidu Group of Teaching Hospital Swat.

Corresponding Author: Hussan Zeb

Email: zebjollity@gmail.com

\section{Assessment of COVID-19 Management at Saidu Group of Teaching Hospital Swat: Analytical Cross-Sectional Study}

\author{
Akhtar Ali Khan ${ }^{1}$, Anwar Ali2, Hussan Zeb3 ${ }^{3}$, Abdul Muneem4, Ijaz Arif5, \\ Bakht Rahman ${ }^{6}$, Misal Khan7, Muhammad Naeem Awan ${ }^{8}$
}

\section{Abstract}

Background: This research aimed to assess the current care management processes for COVID-19 and determine patient outcomes.

Methods: An analytical cross-sectional study was conducted at Saidu group of teaching hospital, a tertiary care hospital with more than a thousand-bed capacity. The target population was all patients admitted to the corona isolation ward and the hospital's critical unit. All the patients admitted through the emergency and inpatient department with Positive PCR, and respiratory symptoms were included in the study.

Results: In total, data of 151 patients were collected. Of these, 90 (59.6\%) were male and 61 (40.4\%) were female with a mean age of $59.17 \pm 13.79$. The most common presenting symptoms were difficulty in breathing $(n=147$, $97.4 \%)$ and dry cough $(n=104,68.9 \%)$, followed by aches and pain $(n=71$, $47 \%)$ and fever $(n=60,39.7 \%)$. Most of the patients had an underlying health condition $(n=96,63.6 \%)$. The most common underlying conditions were hypertension $(n=65,43 \%)$ and diabetes $(n=56,37.1 \%)$. The mean time for the hospital stay was $11.68 \pm 15.31$. About $64 \%$ of the patients recovered with the treatment and care protocols $(n=97,64.2 \%)$, while the remaining $(n=54$, $35.8 \%$ ) died.

Conclusion: This study provides an overview of the clinical presentation and treatment protocol for COVID-19. Most of the patients received supportive treatment comprising of antivirals, steroids, and oxygen therapy. However, the mortality rate was low compared to other hospitals across the country.

Keywords: COVID-19; medical management; nursing care; treatment protocol; health care

\section{Introduction}

$\mathrm{T}$ he coronavirus, referred to as COVID-19 that emerged in Wuhan, spread rapidly across the world and was declared a pandemic. To date (December 20, 2020), there are 81,146,264 confirmed cases, and 1,771,161 deaths due to COVID19 worldwide (1). In Pakistan, the current number of cases is 473,309, with 9,929 fatalities and 423,892 recoveries (2). With the rapid progression of this infection, a greater emphasis has been placed on mass screening, identifying cases in the community, isolating them, and quarantining the cases to prevent further spread (3). However, the health care systems, institutions, and healthcare workers across the highincome countries such as China, Italy, Spain, UK, and the US have also collapsed with the increasing cases of hospital admissions and greater need for quick and timely management $(1,3)$. The COVID-19 also poses a direct threat to the sustainability and challenge to the resilience of the health care system of low-income countries such as Pakistan. 
Globally, the guidelines for the medical and nursing management of patients with COVID-19 in the hospitals are sparse and still being developed. The policies also vary from case to case (i.e., the severity of symptoms) and context to context basis. For example, concerning the severity of symptoms, mild cases of COVID-19 are recommended to self-isolate at homes, moderate cases are managed at homes or within the health care facilities, and severe cases are admitted and managed in intensive care units (4). On the other hand, the management of cases within the health care facilities across countries is quite variable and contingent upon the resources. The general guidelines include patients' assessment in community fever clinics and referring the patient to the health care facilities. In the care facilities, basic infection prevention protocols are followed. These protocols include keeping patients in negative pressure rooms, mandating front-line health care providers to use N95 mask and personal protective equipment when interacting with and caring for the patients, encouraging care providers to perform frequent hand washing before and after interacting the patients, and maintain an appropriate distance of about 6 feet (57).Regarding medical management, various medications and traditional Chinese medicines have been used to treat and manage the symptoms of COVID-19 (8-11). Among the western medicines, antiretroviral used for HIV (Lopinavir / Ritonavir) (10, 12), antivirals for Ebola (remdesivir) and flu (Favipiravir) (13, 14), antimalarial (chloroquine and hydroxychloroquine (15) and anti-rheumatic (Tocilizumab) (16). All of these treatments are currently being tested in various trials such as CATCO (Canadian Treatments for COVID-19) SOLIDARITY trial by WHO, RECOVERY trial in the UK, and DISCOVERY trial in Europe $(15,16)$.

The medical management of COVID-19 is paramount for managing patient symptoms and minimizing the mortality rates. However, equally important is the overall clinical care within health care facilities for early recognition of infected patients, initiating infection prevention and control measures, improving patient prognosis, and providing safe and supportive care for any arising complications (4). A more holistic and comprehensive approach (from admission to discharge) to care for patients with COVID-19 would be beneficial for reducing the work burden of the health care system, institutions, and front-line care providers as well as minimizing the chances of cross- infection. However, no studies have yet been focused on exploring patients' overall management with COVID-19 in health care facilities. Given this, it is essential to assess the processes and strategies used to manage patients with COVID-19 in Pakistan's healthcare facilities. Gaining this knowledge would help develop contextual and tailored guidelines from within the Pakistani context for effective management of patients. The development of contextual and tailored guidelines and their implementation, in turn, would reduce the COVID-19 burden from the health care system and institutions.

\section{Purpose}

To explore the current health care management processes for COVID-19 management and determine patient outcomes.

\section{Methodology}

This analytical cross-sectional study was conducted at Saidu group of teaching hospital, a tertiary care and largest hospital of Malakand division having more than a thousand-bed capacity. The daily OPD patient ratio is from 4,000 to 5,000. For corona management, there were three General wards, each having a 20-bed capacity. One HDU (High Dependency Unit) having 20 bed capacity and one Intensive care Unit having 12 bed. The cross-sectional design was appropriate because the researchers intend to determine the response of patients towards the treatment regime at a single point in time without any intervention.

Target Population and Sample Characteristics

The target population was all patients admitted to the Critical unit at Saidu group of teaching hospital. All the patients admitted through the emergency and inpatient department with Positive PCR and respiratory symptoms were included. A precise estimation for the recruitment of participants was not completed due to inconsistent patient flow. Due to the absence of the sampling frame and an adequate estimated target population, a purposive sampling technique was used on the basis of the following inclusion criteria.

- Patients above 18 years of age

- Patients who were having COVID 19 PCR positive.

- Patients who had been admitted in the hospital for at least 24 hours

\section{Data Collection Instrument}

A structured questionnaire was used for data collection. The questionnaire contains demographic 
data and information regarding signs and symptoms, emergency management, physicians' plan, medical management, vital signs, nursing management, past medical history, shifting notes, isolation precaution notes, discharge, and death summary. The questionnaire was formulated after extensive literature review and discussion with experts.

\section{Data Collection Process}

Data collection lasted from June 2020 to September 2020. All the patients admitted to the unit were included. Consent was taken from the patient and the relative as well. Before data collection, the participants received complete written and verbal

information about the study and were asked for their written consent.

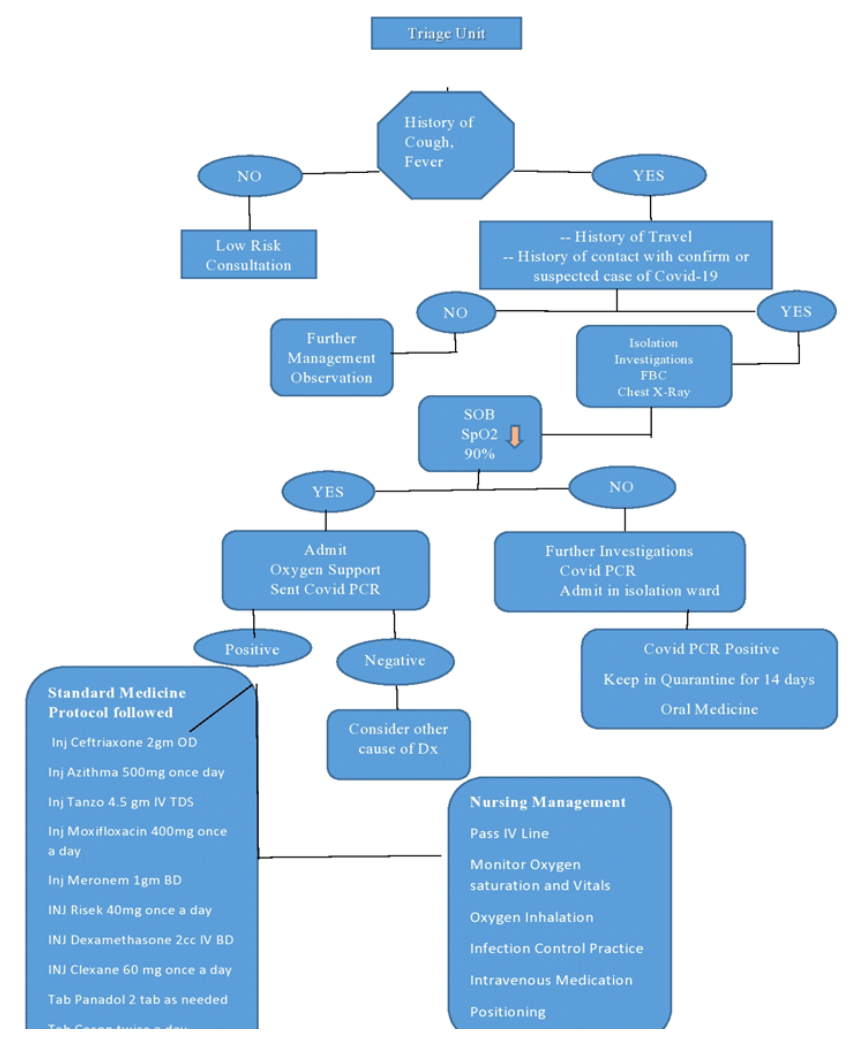

Figure 1. Treatment Protocol

\section{Data Analysis Plan}

The completed questionnaires were checked for errors, coded, and then entered into SPSS (Social Package for the Social Sciences) 24.0 for analysis. The descriptive statistics such as means and standard deviations were calculated for the items.

\section{Ethical Considerations}

The ethical approval for the study was obtained from the Institutional Review Board of Saidu Medical College, Swat, Pakistan. Written permission was also obtained from the management of the hospital. Written informed consent was obtained from the study participants and the attendants. The participants were continuously reminded and informed of their right to review the consent. Protocols were set to maintain the confidentiality and anonymity of the participants

\section{Results}

In total, data were collected for 151 patients. Of these patients, $90(59.6 \%)$ were male and $61(40.4 \%)$ were female with a mean age of $59.17 \pm 13.79$. Most of these patients did not have any educational qualification $(\mathrm{n}=86,57 \%)$ and most of the patients were housewives $(n=60,39.7 \%)$ and labours $(n=36,23.8 \%)$. Most of these patients were received at the emergency care $(n=113$, $74.8 \%$ ), while others were admitted directly to the inpatient units $(n=38,25.2 \%)$. All of these patients received a confirmed diagnosis for COVID-19 ( $n=150$, 99.3\%) except one. None of these patients had any travel history. The demographic data of study participants is summarized in Table 1, along with chi square results.

Table 1. Sociodemographic characteristics of the study population

\begin{tabular}{|l|l|c|c|}
\hline Variables & Categories & Frequency & Percentage\% \\
\hline Sex & Male & 90 & 59.6 \\
& Female & 61 & 40.4 \\
\hline \multirow{2}{*}{ Age } & Under 25 & 2 & 1.3 \\
& $26-35$ & 8 & 5.3 \\
& $36-45$ & 20 & 13.2 \\
& $46-55$ & 26 & 17.2 \\
& Above 55 & 95 & 62.9 \\
\hline \multirow{5}{*}{ Education } & No education & 86 & 56.9 \\
& Primary & 14 & 9.2 \\
& Middle & 18 & 11.9 \\
& Matriculation & 11 & 7.2 \\
& Bachelor's & 16 & 10.5 \\
& Master's & 6 & 3.9 \\
\hline
\end{tabular}




\begin{tabular}{|l|l|c|c|}
\hline Profession & Housewife & 60 & 39.7 \\
& Teacher & 10 & 6.6 \\
& Nurse & 1 & 0.66 \\
& Doctor & 1 & 0.66 \\
& Businessman & 17 & 11.2 \\
& Labor & 36 & 23.84 \\
& Other & 26 & 17.2 \\
\hline “Travel & Yes & 0 & $0 \%$ \\
History" & No & 151 & $100 \%$ \\
\hline
\end{tabular}

Comparison of outcome with disease status and demographics is summarized in table 2

Table 2: Comparison of Outcome with demographic and diseases status. ( $n=151)$

\begin{tabular}{|c|c|c|c|c|}
\hline \multirow[b]{2}{*}{ Variables } & \multirow[b]{2}{*}{ Stratification } & \multicolumn{2}{|c|}{ Outcome } & \multirow{2}{*}{$\begin{array}{c}\mathrm{p}- \\
\text { value }\end{array}$} \\
\hline & & $\begin{array}{c}\text { Deceased } \\
(\mathrm{n}=54)\end{array}$ & $\begin{array}{c}\text { Recovered } \\
(n=97)\end{array}$ & \\
\hline \multirow{4}{*}{ Age, years } & Under 25 & $1(0.66 \%)$ & $1(0.66 \%)$ & \multirow{4}{*}{0.068} \\
\hline & $26-35$ & $2(1.3 \%)$ & $6(3.9 \%)$ & \\
\hline & $36-45$ & $3(1.9 \%)$ & $17(11.2 \%)$ & \\
\hline & $\begin{array}{l}46-55 \\
56 \text { and above }\end{array}$ & $\begin{array}{l}13(8.6 \%) \\
35(23.17 \%)\end{array}$ & $\begin{array}{c}13(8.6) \\
60(39.7 \%)\end{array}$ & \\
\hline \multirow{2}{*}{ Gender } & Male & $32(21.2 \%)$ & $58(38.4 \%)$ & \multirow{2}{*}{0.949} \\
\hline & Female & $22(14.5 \%)$ & $39(25.8 \%)$ & \\
\hline \multirow[b]{2}{*}{ Diabetic } & Yes & $32(21.2 \%)$ & $34(22.5 \%)$ & \multirow[b]{2}{*}{0.488} \\
\hline & No & $22(14.5 \%)$ & $\begin{array}{c}63(41.7 . \\
\%)\end{array}$ & \\
\hline \multirow{2}{*}{ Hypertension } & Yes & $\begin{array}{c}25(16.5 . \\
\%)\end{array}$ & $40(26.4 \%)$ & \multirow{2}{*}{0.547} \\
\hline & No & $29(19.2 \%)$ & $57(37.7 \%)$ & \\
\hline
\end{tabular}

Clinical Presentation and Recovery

The most common presenting symptoms were difficulty in breathing $(\mathrm{n}=147,97.4 \%)$ and dry cough $(\mathrm{n}=104,68.9 \%)$, followed by aches and pain $(\mathrm{n}=71$, $47 \%)$, fever $(n=60,39.7 \%)$, tiredness $(n=53,35.1 \%)$, headache $(n=19,12.6 \%)$, sore throat $(n=12,7.9 \%)$, and diarrhea $(n=04,2.6 \%)$. The mean readings for the vital signs were: blood pressure (Systolic 128.05 \pm 15.63 ), oxygen saturation (73.34 \pm 12.48$)$, temperature (98.68 \pm 4.67$)$, pulse $(102.92 \pm 16.27)$, and respiratory rate (31.20 \pm 10.09$)$. Most of the patients had an underlying health condition $(n=96,63.6 \%)$. The most common underlying conditions were hypertension $(n=65,43 \%)$ and diabetes $(n=56,37.1 \%)$. The mean time for the hospital stay was $11.68 \pm 15.31$. In total, most of the patients recovered with the treatment and care protocols $(\mathrm{n}=97,64.2 \%)$, while the remaining patients died $(n=54,35.8 \%)$. The treatment protocol for COVID-19 is presented in Figure 1.

\section{Discussion}

This study targeted the patients admitted in the Corona Unit from OPD/emergency till the discharge or death. A total of 151 patients were followed, which were admitted in different wards designed for patients with COVID-19. The clinical symptoms mostly observed with covid-19 patients were cough, difficulty in breathing, and sore throat. Our study treatment guidelines after admitting the patient to the ward who meet the admission criteria were intravenous cannulation, oxygen administration, antibiotics for secondary infections, antivirals, and steroids. All the patients were put on the standard treatment protocol. In total, most of the patients recovered with the treatment and care protocols $(n=97,64.2 \%)$, while the remaining patients died $(\mathrm{n}=54,35.8 \%)$. Contrarily, Rahim et al. identified that the general mortality was $77 \%$ of the total admitted patients, and $61.8 \%$ of the patients were kept on non-invasive ventilation (NIV) [17]. The mortality rate for Invasive Mechanical ventilation (IMV) was $93.6 \%$ and $66.7 \%$ was for NonInvasive ventilation $\mathrm{p}<0.001$ ), which is much higher. Grasselli et al, also reported from retrospective case series of 1,591 critically ill patients infected with SARSCoV-2 from Italy, the overall mortality was $26 \%$, which is quite similar to our study mortality (35.8\%) rate (18).

In our study, seven $(4.6 \%)$ patients received plasma therapy, of which four patients died, and three recovered, which indicates that plasma therapy was not helpful in the treatment and recovery. Although the number of patients who received plasma therapy was significantly less, a clinical trial was carried out in China, which compared the two groups. Clinical improvement occurred in $51.9 \%$ in the convalescent plasma group and $43.1 \%$ in the control group, which means there is no significant difference in both groups (19). In this study of 151 admitted patients, 56 (37\%) were diabetic, of which $25(39.28 \%)$ patients died, which is a relatively controlled rate. It is contrary to a study done by Rahim et al. which found that mortality with diabetes mellitus was $(80.0 \%)$, which was highly alarming (17). A study carried out by Sourij et al (20), regarding the fatality of COVID 19 in Austria on patients having diabetes, the finding of the study 


\section{Assessment of COVID-19 Management at Saidu Group of Teaching Hospital Swat: Analytical Cross-Sectional Study}

reported that the mortality of patients with diabetes were $26.7 \%$ comparable to our study which is $39.2 \%$.

There are some limitations of this study such as a small and purposive sample, data collection from a single setting, and limited validity and reliability testing of the data collection form. Further studies should be conducted after consideration of the given limitations.

\section{Conclusion}

This is the first study that provides an overview of the clinical presentation and treatment protocol for COVID-19 in public health care settings. Most of the patient received supportive treatment comprising of antivirals, steroids, and oxygen therapy. However, the mortality rate was low compared to other hospitals across the country. The treatment protocol used in this hospital could be adapted across other hospitals in the country.

\section{References}

1. COVID J. Global Confirmed Cases by Country/Region/Sovereignty: Johns Hopkins University; 2020 [cited 2020 18/03].

2. Government of Pakistan. Covid-19 situation. [Internet]. 2020 [cited 2020 December 20]. Available from: https://covid.gov.pk/

3. World Health Organization. Global surveillance for COVID-19 caused by human infection with COVID-19 virus: interim guidance, 20 March 2020. World Health Organization; 2020.

4. World Health Organization. Rational use of personal protective equipment for coronavirus disease (COVID19): interim guidance, 27 February 2020. World Health Organization; 2020.

5. Wang, H., Wang, S. \& Yu, K. COVID-19 infection epidemic: the medical management strategies in Heilongjiang Province, China. Crit Care 24, 107 (2020). https://doi.org/10.1186/s13054-020-2832-8

6. Marchand-Senécal X, Kozak R, Mubareka S, Salt N, Gubbay JB, Eshaghi A, et al. Diagnosis and management of first case of COVID-19 in Canada: lessons applied from SARS-CoV-1. Clinical Infectious Diseases. 2020 Oct 15;71(16):2207-10. doi.org/10.1093/cid/ciaa227

7. World Health Organization. Rational use of personal protective equipment for coronavirus disease ( COVID19 ( and considerations during severe shortages: interim guidance, 6 April 2020. World Health Organization; 2020.

8. Ren JL, Zhang AH, Wang XJ. Traditional Chinese medicine for COVID-19 treatment. Pharmacol Res. 2020 May; 155: 104743.
9. Runfeng L, Yunlong $\mathrm{H}$, Jicheng $\mathrm{H}$, Weiqi $\mathrm{P}$, Qinhai $\mathrm{M}$, Yongxia $\mathrm{S}$, et al. Lianhuaqingwen exerts anti-viral and anti-inflammatory activity against novel coronavirus (SARS-CoV-2). Pharmacological research. 2020 Jun 1;156:104761.

10. Mahase E. Covid-19: what treatments are being investigated? BMJ 2020; $368 \quad$ :m1252 doi:10.1136/bmj.m1252

11. Huang C, Wang Y, Li X, Ren L, Zhao J, Hu Y, et al. Clinical features of patients infected with 2019 novel coronavirus in Wuhan, China. The lancet. 2020 Feb 15;395(10223):497-506. doi.org/10.1016/S01406736(20)30183-5

12. Sheahan TP, Sims AC, Graham RL, Menachery VD, Gralinski LE, Case JB, et al. Broad-spectrum antiviral GS-5734 inhibits both epidemic and zoonotic coronaviruses. Science translational medicine. 2017 Jun 28;9(396). DOI: 10.1126/scitranslmed.aal3653

13. Holshue ML, DeBolt C, Lindquist S, Lofy KH, Wiesman J, Bruce H, et al. First case of 2019 novel coronavirus in the United States. N Engl J Med 2020; 382:929-936. DOI: 10.1056/NEJMoa2001191

14. Blueprint RD. COVID-19. World Health Organization. 2020.Clinical Trials Arena (2020).

15. Covid-19 trials begin in the UK, Canada and Europe. https://www.clinicaltrialsarena.com/news/covid-19trial-uk-canada-europe/

16. Cheng MP, Lee TC, Tan DH, Murthy S. Generating randomized trial evidence to optimize treatment in the COVID-19 pandemic. CMAJ. 2020 Apr 14;192(15):E4057.

17. Rahim F, Amin S, Noor M, Bahadur S, Gul H, Mahmood A, et al. Mortality of Patients With Severe COVID-19 in the Intensive Care Unit: An Observational Study From a Major COVID-19 Receiving Hospital. Cureus. 2020 Oct;12(10). doi: 10.7759/cureus.10906

18. Grasselli G, Zangrillo A, Zanella A, Antonelli M, Cabrini L, Castelli A, et al. Baseline characteristics and outcomes of 1591 patients infected with SARS-CoV-2 admitted to ICUs of the Lombardy Region, Italy. Jama. 2020 Apr 28;323(16):1574-81. doi:10.1001/jama.2020.5394

19. Li L, Zhang W, Hu Y, Tong X, Zheng S, Yang J, et al. Effect of convalescent plasma therapy on time to clinical improvement in patients with severe and lifethreatening COVID-19: a randomized clinical trial. Jama. $2020 \quad$ Aug 4;324(5):460-70. doi:10.1001/jama.2020.10044

20. Sourij H, Aziz F, Bräuer A, Ciardi C, Clodi M, Fasching $\mathrm{P}$, et al. COVID-19 fatality prediction in people with diabetes and prediabetes using a simple score upon hospital admission. Diabetes, Obesity and Metabolism. 2021 Feb;23(2):589-98. doi.org/10.1111/dom.14256 